\title{
A Co-Axial Microtubular Flow Battery Cell with Ultra-high Volumetric Power Density
}

Yutong Wu ${ }^{1, \downarrow}$, Fengyi Zhang ${ }^{1, \downarrow}$, Ting Wang ${ }^{2,+}$, Po-Wei Huang ${ }^{1}$, Haochen Yang ${ }^{1}$, Alexandros Filippas ${ }^{1}$, Yanghang Huang ${ }^{1}$, Chao Wang ${ }^{1}$, Huitian Liu ${ }^{1}$, Xing Xie ${ }^{2} *$, Ryan P. Lively ${ }^{1} *$ and Nian $\operatorname{Liu}^{1, *}$

${ }^{1}$ School of Chemical and Biomolecular Engineering, Georgia Institute of Technology; Atlanta, GA 30332, USA.

${ }^{2}$ School of Civil and Environmental Engineering, Georgia Institute of Technology; Atlanta, GA 30332, USA.

tThese authors contributed equally to this work.

*Correspondence to: xing.xie@ cee.gatech.edu (X. X.); ryan.lively@ chbe.gatech.edu (R. P. L.); nian.liu@ chbe.gatech.edu (N. L.)

\begin{abstract}
Volumetric power density is a key factor determining flow batteries' footprint, capital cost and ability to handle uneven energy resource distributions. While significant progress has been made on flow battery materials and electrochemistry to improve energy density, conventional flow battery assemblies based on planar cell configuration exhibit low packing efficiencies and membrane surface area per volume of the cell, thus resulting in low volumetric power density. Here, we introduce a co-axial microtubular (CAMT) flow battery cell that significantly improves the volumetric power density. This cell design overcomes the intrinsic power limit of planar cell configuration and is suitable for all mainstream flow battery chemistries. Using zinc-iodide chemistry as a demonstration, our CAMT cell shows peak charge and discharge power densities of $1322 \mathrm{~W} / \mathrm{L}_{\text {cell }}$ and $306.1 \mathrm{~W} / \mathrm{L}_{\text {cell }}$ compared to average charge and discharge power densities of < $60 \mathrm{~W} / \mathrm{L}_{\text {cell }}$ and $45 \mathrm{~W} / \mathrm{L}_{\text {cell }}$ of conventional planar flow battery cells. In addition, the battery can cycle for more than 220 hours, corresponding to $>2,500$ cycles at off-peak conditions. Furthermore, we have also demonstrated that the CAMT cell is compatible with zinc-bromide, quinone-bromide, and all-vanadium chemistries. The CAMT flow cell represents a device-level innovation to enhance the volumetric power of flow batteries, and potentially reduce the size and cost of the cells and the entire flow battery. The CAMT design can potentially be applied to other electrochemical systems and lead to a paradigm shift in flow battery fundamental study and commercialization.
\end{abstract}




\section{Broader Context}

Renewable energy sources such as wind, solar, and biomass can potentially out-weight unsustainable fossil fuels and eventually lead to a zero-emission society. Due to the uneven energy resource distribution and time-dependent fluctuation in energy generation, the harvest, storage, and delivery of renewable energy are crucial to match the enormous demand. Flow batteries are a promising technology for large-scale and long-duration energy storage; they exhibit unparalleled advantages in scalability, design flexibility, and lifespan because of the spatially decoupled energy storage and power conversion units. However, flow batteries today are still based on cell configurations developed decades ago. The planar cell configuration exhibit low packing efficiencies and membrane surface area per volume of the cell, thus resulting in low volumetric power density, high footprint and capital cost. An alternative overall cell is necessary to achieve a higher volumetric power density, decrease the capital cost and increase the competitiveness of flow batteries relative to alternative technologies. 
Renewable energy sources are driving a global energy transition towards a zero-emission society 1,2 , and grid-scale energy storage technologies are needed to address uneven resource distributions and energy fluctuations. Volumetric power density is a key factor determining energy storage technologies' footprint and capital cost. Unlike traditional secondary battery systems using solid active materials, flow batteries decouple energy storage (i.e., the concentration of electrolyte and storage container size) and power conversion (i.e., the central electrochemical reaction energy conversion device), featuring high safety and long battery life ${ }^{3-6}$. The central cell unit where redox active species receive or lose electrons mainly consists of two electrodes, flow distributors, and a planar ion exchange membrane (Fig. 1a); these components are costly and occupy most of the cell volume. The capital cost of flow batteries $(\sim 800 \mathrm{US} \$ / \mathrm{kWh})^{7}$ is significantly higher than Li-ion batteries $(<300 \mathrm{US} \$ / \mathrm{kWh}){ }^{8}$ and far from the $125 \mathrm{US} \$ / \mathrm{kWh}$ goal set by the United States Department of Energy ${ }^{1}$. Also, typical flow batteries have a volumetric power density of $<100$ $\mathrm{W} / \mathrm{L}_{\text {cell }}{ }^{9}$, while Li-ion batteries have power densities $\sim 1500 \mathrm{~W} / \mathrm{L}_{\text {cell }}{ }^{10,11}$, flow batteries are thus not widely deployed because of their low volumetric power density and high capital cost ${ }^{12-14}$. It is of great interest to increase the cell volumetric power density since it significantly impacts the competitiveness of flow batteries relative to alternative technologies ${ }^{14}$.

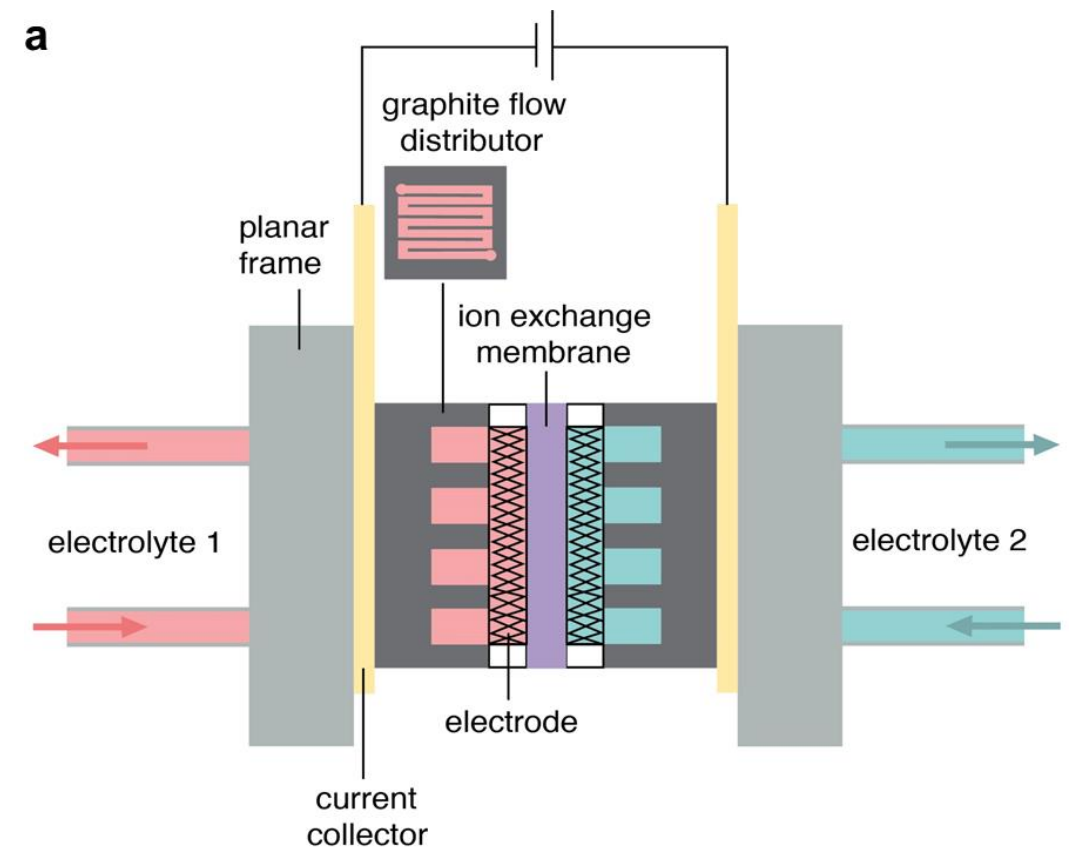

b

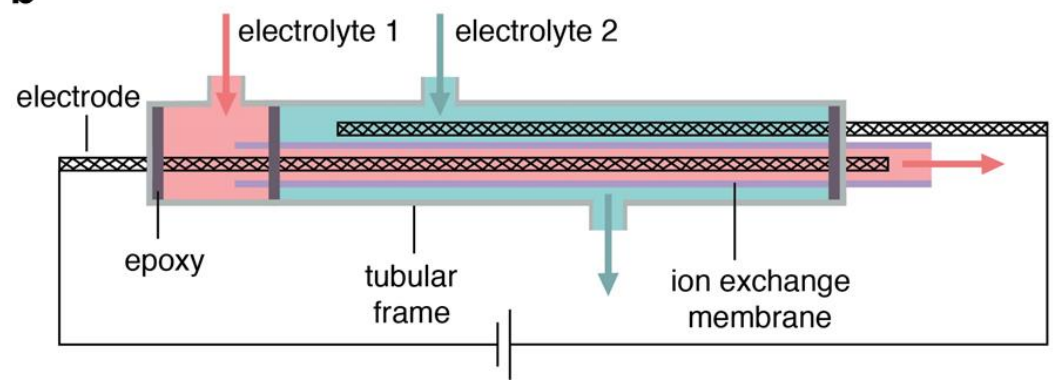

Fig. 1 Comparison between the conventional flow battery cell with a planar membrane and the coaxial microtubular (CAMT) flow battery cell design. (a) Conventional planar cell design. (b) CAMT cell design. 
While significant progress has been made in advanced materials and chemistry based on the conventional planar cell geometry, limited research has been conducted at the device level ${ }^{6,15}$. An alternative overall cell design with improved packing efficiency and membrane surface-area-tovolume ratio is necessary to achieve a higher volumetric power density and decrease the size of the cell. In the chemical separation industry, hollow fiber membrane modules are known as the most space-efficient form of membrane device. For instance, industry-scale hollow fiber membrane modules provide around $10,000 \mathrm{~m}^{2}$ surface area per $1 \mathrm{~m}^{3}$ module, which is more than an order of magnitude higher than traditional planar-style membrane modules ${ }^{16}$ (e.g., spiralwound membrane module). The tubular membrane geometry compensates the transmembrane pressure via the compression of membrane materials, allowing continuous operation under pressurized conditions without additional supporting materials such as spacers and fabric backing. This highly scalable configuration has been proven effective at reducing the membrane device footprint (via increases in device productivity, i.e., moles of product per second per cubic meter of device) in various separation processes such as gas separation, liquid-phase separation, and organic solvent separations ${ }^{16,17}$. Attempts to use hollow fibers have also been made in solid oxide fuel cells ${ }^{18}$.

Herein, we report a co-axial microtubular (CAMT) flow battery cell design to eliminate the intrinsic limits of the planar membrane cell design and achieve an order of magnitude increase in volumetric power density for flow batteries (Fig. 1b). In the CAMT cell, co-flowing electrolytes pass through the device from bore and shell sides and are separated by microtubular ion-exchange membranes (e.g. Nafion) while the electrodes extend out from the center. There are several advantages of this design. First, the membrane surface-area-to-volume ratio is increased to match that of the electrode surface area, so the membrane no longer limits the ion transfer. Second, the microtube itself serves as the electrolyte distributor without any other supporting materials, avoiding extra distribution apparatus that decrease volumetric power density. Third, the system packing efficiency is increased to have a smaller footprint at the same power level. All the components used to fabricate the CAMT cell in this work are off-the-shelf and the scaling up can follow the hollow fiber modules in the chemical separation industry ${ }^{19}$.

The zinc-iodide redox system (reactions shown in Fig. 2a) was chosen to demonstrate the concept of the CAMT flow battery because of its high theoretical energy density of up to $322 \mathrm{Wh} /$ Lelectrolyte, and ability to operate in ambient air ${ }^{4,20,21}$. Carbon fiber electrodes were inserted into the bore sides of Nafion microtubular membranes $(0.65 \mathrm{~mm}$ OD) to serve as the cathode for the iodide half reaction, and a zinc wire $(0.25 \mathrm{~mm}$ OD) was placed in the shell side outside the membranes to serve as the anode for the zinc half reaction (Fig. 2a). The numbers of positive and negative electrodes in a cell can be adjusted to balance the reaction kinetics of two half reactions and maximize the volumetric power density. Fig. $2 b$ and Fig. S1 show the cross-sectional scanning electron microscopy (SEM) images of the co-axial microtubular cell. As shown in Fig. 2c, zinciodide flow batteries with CAMT cells were built and used for performance evaluation experiments. The electrolytes flow in the same direction during operation. The effective working length of the CAMT cell, where the reaction occurred, was $5 \mathrm{~cm}$. The length was increased to $8 \mathrm{~cm}$ in Fig. $2 \mathrm{a}$ for demonstration purposes and can potentially be further increased. Fig. S2 compares the CAMT module to a typical lab-scale planar cell module. The thick battery frame under pressure during operation to hold the cell components and extra flow distributors are no longer needed for the CAMT design. 

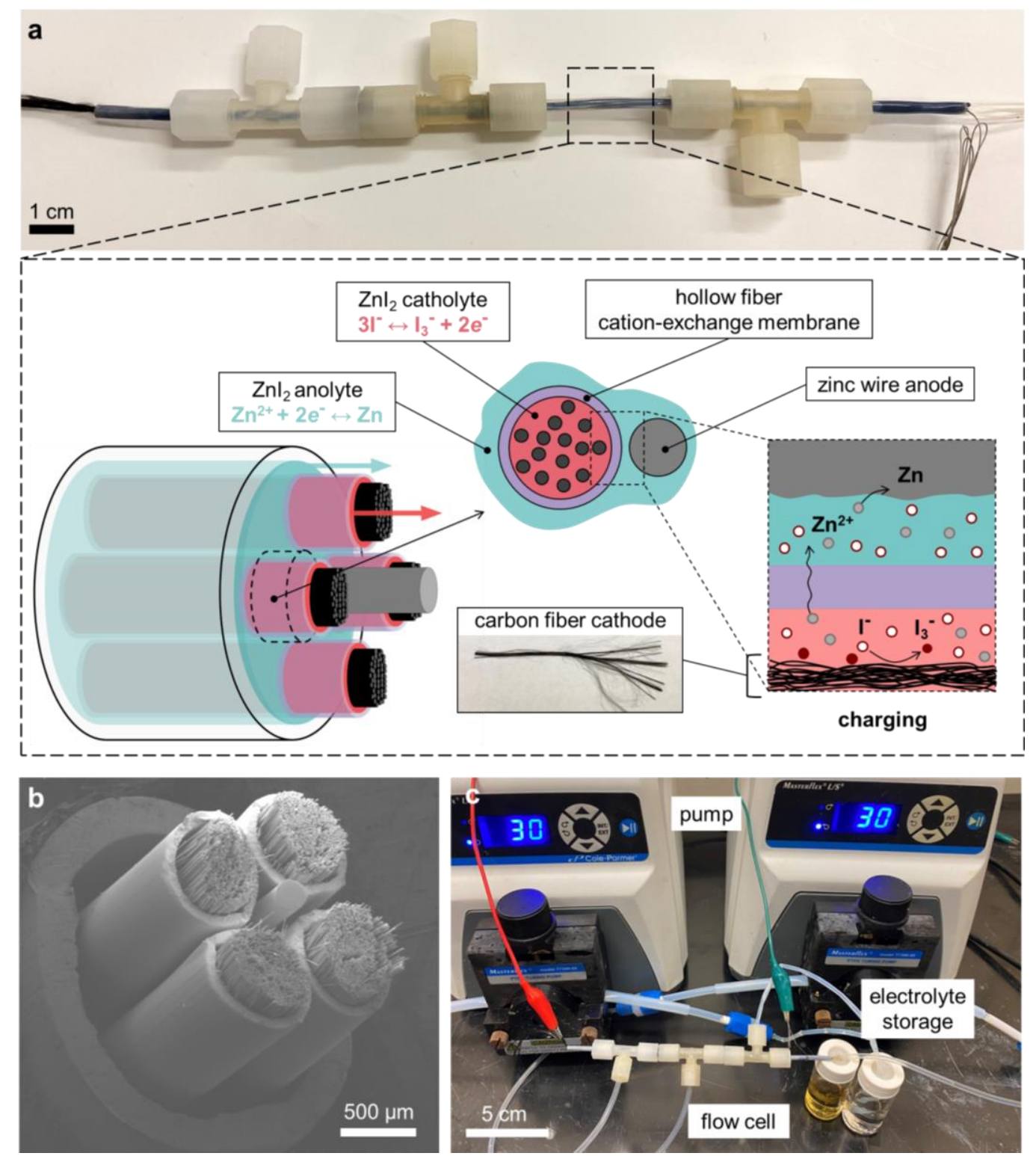

Fig. 2 CAMT flow battery using zinc-iodide redox as a demonstration. (a) On the top is a picture of a zinc-iodide CAMT module with four positive electrodes and one negative electrode; on the bottom is an illustration of its detailed structure and the electrochemical reactions during charging. (b) A cross-sectional SEM image of the zinc-iodide CAMT module. (c) Experimental setup of the CAMT flow battery.

Electrochemical Impedance Spectroscopy (EIS) was conducted to confirm the ion-conduction function of the commercial Nafion microtubes. First, carbon fiber and Zn wire electrodes of the same total length as those used in the microtubular cell were inserted into two $2 \mathrm{M} \mathrm{ZnI}_{2}$ electrolyte containing H-shaped electrolytic cells (H-cells). For one H-cell, a planar Nafion 117 membrane was sandwiched in the middle and for the other $\mathrm{H}$-cell, a Nafion microtubular membrane was enclosing one of the carbon fiber electrodes, and the two electrodes were $4 \mathrm{~cm}$ effective in length and $5 \mathrm{~cm}$ apart in both H-cells. Fig. 3a shows that the impedance of the Nafion microtubular cell and Nafion 117 membrane cell was of the same magnitude, indicating that the microtube was capable of being used as an ion exchange membrane for the cell. Note that we also compared the 
impedance to an H-cell without a membrane and the impedance was also close to the two samples with membrane, indicating that the electrode-to-electrode distance is significant for a cell to have low impedance. On the other hand, two microtubular cell modules with one and four Nafion microtubes inside were compared to the H-cell results. The impedance for the microtubular cell with one microtube was significantly lower compared with $\mathrm{H}$-cell results because of close packing and decreased electrode-to-electrode distance. The impedance was further reduced for the microtubular battery with four microtubes because of the higher electrode area.

The effect of cell geometry on membrane surface-area-to-volume ratio and overall kinetics was evaluated using finite element simulation, to understand why the close packing microtubular geometry has a higher volumetric power density. We proposed a performance evaluation basis named active volume (electrode area multiplied by the necessary thickness of the cell, unit: per $\left.\mathrm{L}_{\text {cell }}\right)$ as an alternative power density comparison instead of the conventional method based on electrode surface area or electrolyte volume. Active volume is the effective volume necessary for a flow battery cell to achieve its ascribed performance; cell parts such as flow distributor are accounted for since they are indispensable for the flow battery to overcome pressure and transport issues and achieve high performance. Using electrode surface area as a reference overestimates power density, while using electrolyte volume also leaves uncertainty because the volume used depends on the battery geometry and can vary in a wide range based on different experimental purposes. The dimensions of microtubular and planar cell geometries were chosen to reflect realistic cell volumes (Fig. 3b, Table S1). Within the same volume of the cells, the surface area of the membrane in the microtubular cell was 15 times larger than the one in the planar cell. For clarity purposes, the carbon electrodes are not shown in explicit form in Fig. 3b. The current densities at a series of states of charges (SOCs) and cell voltages were calculated and compared. At $75 \%$ SOC, for example, the current densities of the microtubular cell were $\sim 5$ times higher than the ones of the planar cell, at all cell voltages (Fig. 3c, Table S2). A > 5 folds improvement in current density was observed in CAMT compared to the planar configuration for the same redox chemistry, which was consistent with the improvements in mass transfer area. It was also found that the current densities will in general be enhanced by the increase of redox reaction rate constant. At the same reaction rate constant, the current density of the microtubular geometry was always higher than that of the planar geometry because of the enhanced electrode and membrane surface area (Fig. 3d, Table S3). To note that unlike realistic flow batteries, the flow distributor was not accounted for in this idealized scenario and involving it in the simulation would further increase the volumetric power advantage of the CAMT configuration relative to planar cells. 

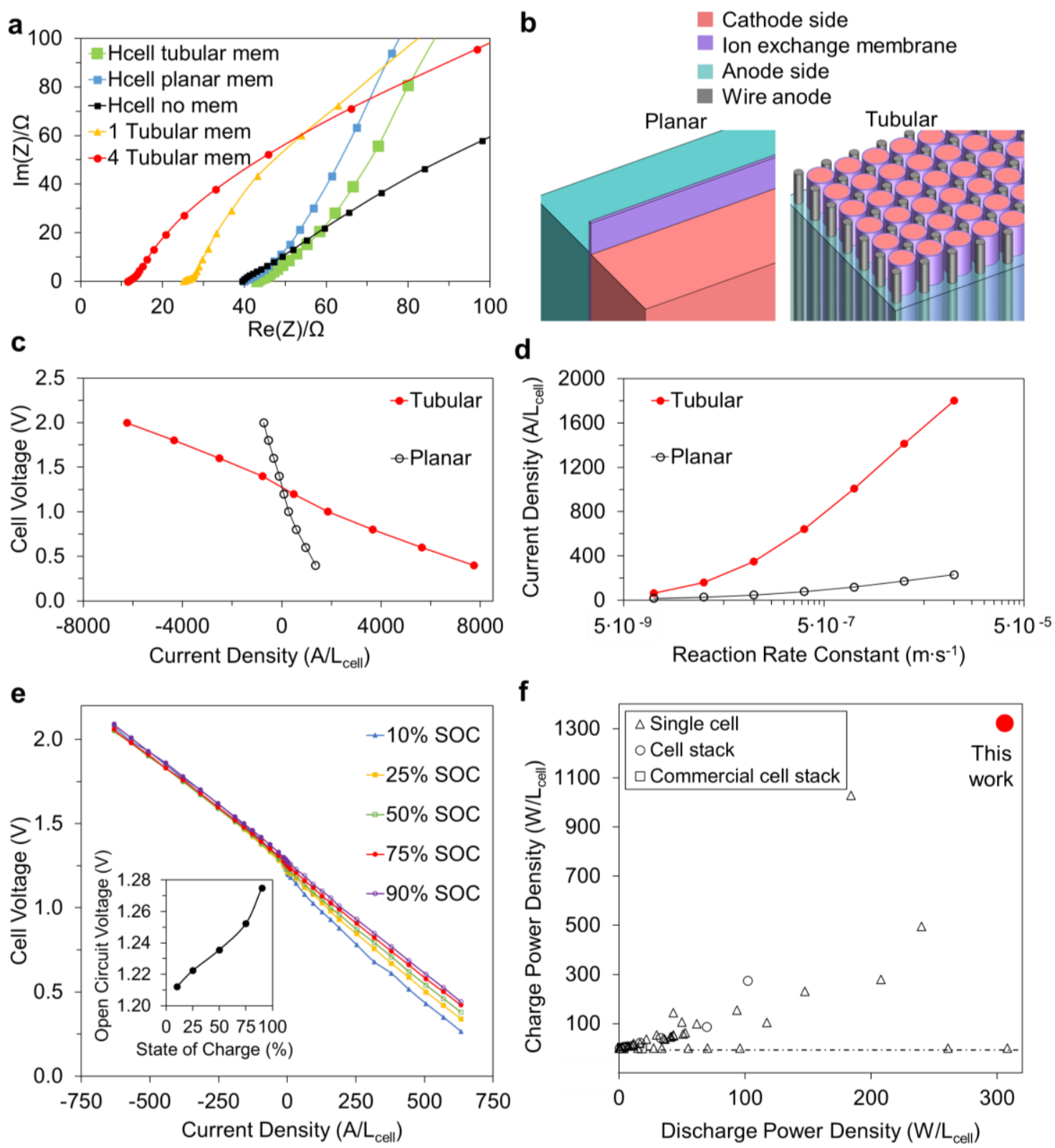

Fig. 3 Experimental and simulated power density of zinc iodide co-axial microtubular cell. (a) comparison of the electrochemical impedance among CAMT cells with i) one and ii) four Nafion microtubes, H-cells iii) without membrane, iv) with a planar Nafion 117 membrane, and v) a Nafion microtubular membrane enclosing one of the electrodes. (b) Finite element simulation models of a closely packed CAMT cell stack and a planar cell stack with the same volume. Carbon electrodes are not shown for clarity of presentation. (c) Simulated current density of microtubular vs. planar cells at a series of cell voltages. (d) Simulated current density of microtubular vs. planar cells at a series of redox reaction rate constants. (e) Experimental microtubular cell voltage vs. volumetric current density at five different SOCs, the electrolyte was $2 \mathrm{M} \mathrm{ZnI}_{2}$ and the higher and lower cut-off voltages were 2.1 and $0.25 \mathrm{~V}$. The inset shows the open circuit voltages at different states of charge. (f) Comparison of maximum charge power density vs. maximum discharge power density of literature values. For the literature that did not report volumetric power, their areal power was converted to volumetric one based on the active volume of their cells. Volumetric power density is recalculated based solely on the information provided from the references. 
The measured electrochemical performance of the zinc-iodide flow battery with the co-axial microtubular cell also supported the hypothesis that a microtubular cell design significantly enhances the volumetric power density. Fig. 3e shows the co-axial microtubular cell voltage vs. volumetric current density at different SOCs. As the SOC increased, the open circuit voltage increased from $1.21 \mathrm{~V}$ to $1.27 \mathrm{~V}$, in the range of a typical zinc-iodide redox couple. The device was able to sustain a current density as high as $632.4 \mathrm{~A} / \mathrm{L}_{\text {cell }}$ during charging and discharging. At 632.4 A/Lcell, the volumetric power density reached $1322 \mathrm{~W} / \mathrm{L}_{\text {cell, }}$ in charging and $306.1 \mathrm{~W} / \mathrm{L}_{\text {cell }}$ in discharging. Based on the total inner surface area of microtubular membranes, the corresponding areal power density were $51.2 \mathrm{~mW} / \mathrm{cm}^{2}$ in charging and $10.8 \mathrm{~mW} / \mathrm{cm}^{2}$ in discharging. Fig. S3 shows the current density vs. power density at different SOCs. When comparing the volumenormalized maximum charge and discharge power density to 47 research articles and 3 commercial flow batteries references using various redox couples (Fig. 3f, Table S5), the performance was significantly higher than most of the references with charge/discharge volumetric power density lower than $60 / 45 \mathrm{~W} / \mathrm{L}_{\text {cell. }}$. For those literatures that did not directly report volumetric power, their areal power was converted to volumetric one based on the active volume of their cells. The above experimental results confirm the hypothesis and numerical simulation that the co-axial microtubular cell with increased membrane surface-area-to-volume ratio and packing efficiency increase the volumetric power density compared to the planar cell.

The zinc-iodide CAMT flow battery was deeply charged to $50 \%$ total capacity and fully discharged using a current of $253 \mathrm{~A} / \mathrm{L}_{\text {cell }}$ as shown in Fig. 4a, achieving a Coulombic efficiency (CE) of 95.8\%. The deep cycling performance proved that the microtubular follow battery can discharge at an ultra-high volumetric power density in a standard commercial flow battery operation time range (4 - 12 hours) ${ }^{14}$. Maintaining a long and stable battery life during the off-peak period is another significant character of flow batteries. A long duration battery test was performed using $1.25 \%$ zinc wire capacity, and the battery was able to run for $>2,500$ cycles, corresponding to more than 220 hours at $31.6 \mathrm{~A} / \mathrm{L}_{\text {cell }}$ (Fig. 4b). Fig. S4 shows the zoomed-in view of the voltage profile after 1,664 cycles. The voltage profile was still steady at the late cycling stage, indicating that the microtubular flow battery can operate stably and safely during off-peak hours. We believe that using advanced materials and chemistry will further increase the volumetric power density. The packing efficiency can also be optimized to enhance the volumetric power density.

Importantly, the microtubular cell configuration is universal and can be applied to other redox couples. Besides zinc-iodide (Movie S1), the CAMT cell configuration were tested with other prominent redox couples. As shown in Fig. 4c, zinc-bromide electrolyte was cycled steadily for 100 cycles at $6.32 \mathrm{~A} / \mathrm{L}_{\text {cell }}$ in CAMT cell. The voltage was steady around the $1.8 \mathrm{~V}$ thermodynamic voltage. Quinone is a popular chemical for organic flow batteries because of its high solubility and ability to be modified by functional groups. As shown in Fig. 4d, 9,10-anthraquinone-2,7disulphonic acid (AQDS) was selected as the anode electrolyte and paired with bromine catholyte and cycled 100 times in CAMT cell. The zinc wire electrode was switched to carbon fiber to avoid side reactions. The voltage fell in the typical cycling range (less than $1.2 \mathrm{~V}$ ). As shown in Fig. 4e, vanadium redox was also cycled in CAMT cell for 100 times as the other two redox couples and showed a steady cycling performance. Therefore, the CAMT flow cell is compatible with various flow battery and hybrid flow battery chemistries. The cell components can be modified to adapt other redox couples such as size-exclusion based polymer electrolytes ${ }^{19}$. 

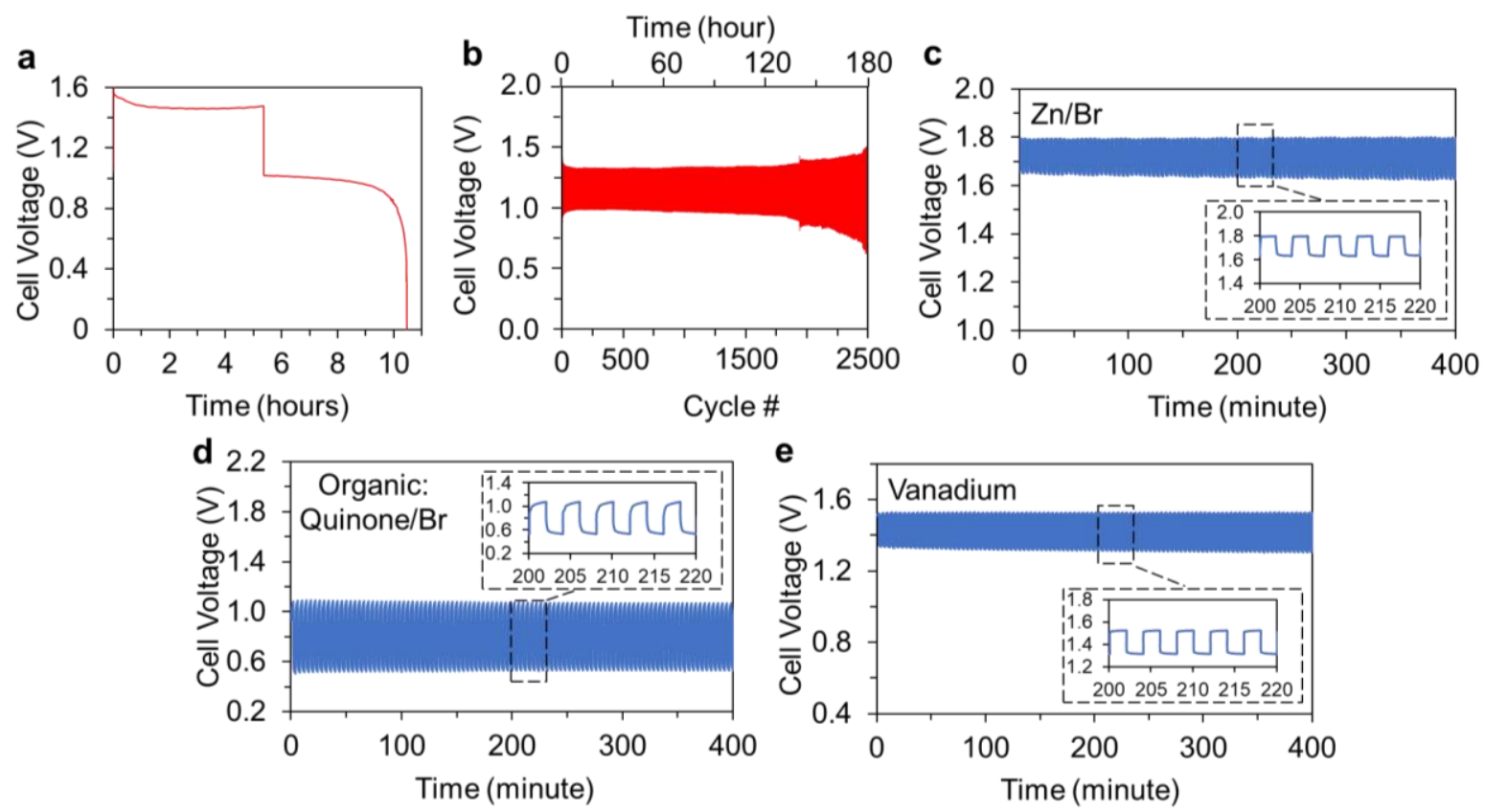

Fig. 4 Zinc-iodide flow battery cycling performance and CAMT cell configuration tested with other redox couples. (a) Charging and discharging voltage profile of the zinc-iodide flow battery cycled between $0 \%$ and $50 \% \mathrm{SOC}$ at $253 \mathrm{~A} / \mathrm{L}_{\text {cell }}$ with $2 \mathrm{M} \mathrm{ZnI}_{2}$ as the initial catholyte and anolyte, with the higher cut-off voltage being $1.65 \mathrm{~V}$. (b) Long-term cycling of the zinc-iodide microtubular cell using $1.25 \%$ zinc wire capacity at $31.6 \mathrm{~A} / \mathrm{L}_{\text {cell }}$, with the higher and lower cut-off voltages being 1.5 and 0.5 V. (c) Cycling of zinc-bromide redox couple, with the higher cut-off voltage being $2 \mathrm{~V}$. (d) Cycling of organic quinone electrolyte vs. $\mathrm{Br}_{2}$, with the higher cut-off voltage being $1.5 \mathrm{~V}$. (e) Cycling of vanadium redox couple, with the higher cut-off voltage being $1.6 \mathrm{~V}$. The three redox couples were cycled at $6.32 \mathrm{~A} / \mathrm{L}_{\text {cell }}$ for 100 times.

The CAMT cells in this work were all assembled from off-the-shelf components. It is difficult to compare the cost of different cell configurations at lab-scale, so we simply list the cost of our flow battery cell module for reference. The material cost of building our lab-scale microtubular cell as shown in Fig. 2a was only $\$ 41.85$ (Table S6), and the cost can be further reduced for the other three redox systems since the $\mathrm{Zn}$ wire will be replaced by carbon fiber. The carbon fiber electrode in our module is the same one that is widely used for pultrusion and enforcement purposes, thus the cost is only $\$ 0.02$ for a single CAMT module containing four fibers. It is important to note that the co-axial flow battery enclosure and ion exchange membrane are the most expensive components, which is also the case in a planar flow battery cell. The cost of Nafion microtubes can potentially be reduced with process innovation and scale up.

The components of CAMT module enclosures can be easily tailored to work with various redox couples and battery sizes. The mature hollow fiber design and fabrication techniques can shape both melt-processable materials and solution-processable materials into hollow fibers, which allows the utilization of a wide range of membrane materials including anion exchange membranes. The modular design of hollow fiber membrane contactor allows easy scale-up and customization for energy storage applications at different scales ${ }^{19,22}$.

In summary, we developed a co-axial microtubular flow battery cell, which increased the packing efficiency and membrane surface-area-to-volume ratio and led to an order of magnitude increase 
in volumetric power density compared to the conventional planar cell configuration. Using zinciodide chemistry as a demonstration, simulation results indicated a $>5$ folds improvement in current density in CAMT compared to the planar configuration for the same redox chemistry, which was consistent with the improvements in mass transfer area. Experimentally, our CAMT battery showed peak charge and discharge power densities of $1322 \mathrm{~W} / \mathrm{L}_{\text {cell }}$ and $306.1 \mathrm{~W} / \mathrm{L}_{\text {cell }}$ compared to average charge and discharge power densities of $<60 \mathrm{~W} / \mathrm{L}_{\text {cell }}$ and $45 \mathrm{~W} / \mathrm{L}_{\text {cell }}$ of conventional planar flow batteries. In addition, the battery was able to cycle for more than 220 hours, corresponding to $>2,500$ cycles during off-peak period. This CAMT cell configuration is also broadly applicable to all flow battery or hybrid flow battery redox couples. We have demonstrated steady long cycle performance for zinc-bromide, quinone-bromide and all-vanadium redox couples. The CAMT cell configuration is expected to be highly scalable following the successful examples in separation industry. It can potentially enhance the performance and reduce the cost of flow batteries, accelerating their market adoption. The CAMT design can potentially be applied to other energy storage systems such as fuel cells and electrolysis and provoke more device-level innovations in emerging electrochemical technologies.

Acknowledgements The authors thank William J. Koros for recommending commercial Nafion The authors thank William J. Koros for recommending commercial Nafion hollow fiber for battery assembly and acknowledge Bryce Smith, Panyiming Liu, Chihyun Hwang and Maxim Zhelyabovskiy for assisting the experiments. Y.W. thanks Kaiyue Yang for figure designing and Wade Wagner for manuscript language revision. Funding N.L. acknowledges support from faculty startup funds from the Georgia Institute of Technology. Characterizations were conducted in part at the Georgia Tech Institute for Electronics and Nanotechnology, a member of the National Nanotechnology Coordinated Infrastructure supported by the National Science Foundation (Grant ECCS-2025462). Author contributions N.L. and Y.W. formed the initial concept of CAMT flow battery cell. Y.W., N.L., F.Z., and R.P.L. designed experiments to demonstrate the concept. Y.W., T.W., H. Y. and X. X. did simulation and calculation. Y.W., P.-W.H. and Y. H. contributed to the early hollow fiber testing and SOC tests. Y.W., A. F., and C.W. built and tested the CAMT cell with different redox couples. Y.W., P.-W.H. and H. L. conducted the characterization experiments and designed the schematics. Y. W., F. Z. and T. W. wrote the first draft of the manuscript. N. L., R. P. L. and X. X. revised the manuscript and provided guidance. Competing interests N.L., Y.W., R.P.L. and F.Z. hold a Patent Cooperation Treaty application WO/2021/076548 through Georgia Tech Research Corporation. Data and materials availability All data are available in the manuscript or in the supplementary materials.

\section{Keywords}

Flow Battery; Energy Storage; High Power; Tubular Reactor; Hollow Fiber Membrane 


\section{References}

1. S. Chu, Y. Cui and N. Liu, Nat Mater, 2016, 16, 16-22.

2. U.S. Renewable Energy Factsheet, Center for Sustainable Systems, University of Michigan, 2020.

3. J. F. Weaver, World's largest battery: 200MW/800MWh vanadium flow battery - site work ongoing, 2017.

4. C. Xie, Y. Liu, W. Lu, H. Zhang and X. Li, Energy \& Environmental Science, 2019, 12, 1834-1839.

5. P. Xiong, L. Zhang, Y. Chen, S. Peng and G. Yu, Angew Chem Int Ed Engl, 2021, DOI: 10.1002/anie.202105619.

6. $\quad$ S. Gu, K. Gong, E. Z. Yan and Y. Yan, Energy Environ. Sci., 2014, 7, 2986-2998.

7. R. F. Service, Science, 2014, 344, 352-354.

8. O. Schmidt, A. Hawkes, A. Gambhir and I. Staffell, Nature Energy, 2017, 2.

9. M. R. Mohamed, S. M. Sharkh and F. C. Walsh, IEEE Vehicle Power and Propulsion Conference, 2009, DOI: 10.1109/VPPC.2009.5289801, 551-557.

10. Battery requirements for future automotive applications, EUCAR, 2019.

11. Advanced Battery Development, DOE Energy Storage R\&D, 2013.

12. B. Huskinson, M. P. Marshak, C. Suh, S. Er, M. R. Gerhardt, C. J. Galvin, X. Chen, A. Aspuru-Guzik, R. G. Gordon and M. J. Aziz, Nature, 2014, 505, 195-198.

13. M. Park, J. Ryu, W. Wang and J. Cho, Nature Reviews Materials, 2016, 2.

14. M. L. Perry, R. M. Darling and R. J. E. T. Zaffou, 2013, 53, 7.

15. X. Li, H. Zhang, Z. Mai, H. Zhang and I. Vankelecom, Energy \& Environmental Science, 2011, 4.

16. R. P. Lively, M. E. Dose, L. Xu, J. T. Vaughn, J. R. Johnson, J. A. Thompson, K. Zhang, M. E. Lydon, J.-S. Lee, L. Liu, Z. Hu, O. Karvan, M. J. Realff and W. J. Koros, Journal of Membrane Science, 2012, 423-424, 302-313.

17. Y. Ma, F. Zhang and R. P. Lively, in Sustainable Nanoscale Engineering, 2020, DOI: 10.1016/b978-0-12-814681-1.00003-5, pp. 33-81.

18. T. Liu, C. Ren, S. Fang, Y. Wang and F. Chen, ACS Appl Mater Interfaces, 2014, 6, 1885318860.

19. C. F. Wan, T. Yang, G. G. Lipscomb, D. J. Stookey and T.-S. Chung, Journal of Membrane Science, 2017, 538, 96-107.

20. B. Li, Z. Nie, M. Vijayakumar, G. Li, J. Liu, V. Sprenkle and W. Wang, Nat Commun, 2015, 6, 6303.

21. H. Pan, B. Li, D. Mei, Z. Nie, Y. Shao, G. Li, X. S. Li, K. S. Han, K. T. Mueller, V. Sprenkle and J. Liu, ACS Energy Letters, 2017, 2, 2674-2680.

22. N. C. Mat, Y. Lou and G. G. Lipscomb, Current Opinion in Chemical Engineering, 2014, 4, 18-24. 